Meta

Journal des traducteurs

Translators' Journal

\title{
La formation d'interprètes : la méthode cognitive
}

\section{Sylvie Lambert}

Volume 34, numéro 4, décembre 1989

URI : https://id.erudit.org/iderudit/003379ar

DOI : https://doi.org/10.7202/003379ar

Aller au sommaire du numéro

Éditeur(s)

Les Presses de l'Université de Montréal

ISSN

0026-0452 (imprimé)

1492-1421 (numérique)

Découvrir la revue

Citer cet article

Lambert, S. (1989). La formation d'interprètes : la méthode cognitive. Meta, 34(4), 736-744. https://doi.org/10.7202/003379ar d'utilisation que vous pouvez consulter en ligne.

https://apropos.erudit.org/fr/usagers/politique-dutilisation/ 


\section{LA FORMATION D'INTERPRÈTES : LA METHODE COGNITIVE}

SYLVIE LAMBERT

Université d'Ottawa, Ottawa, Ontario, Canada

Dans cet article, je décrirai la façon dont j'ai structuré mon séminaire d'initiation à l'interprétation simultanée. De plus, mon intention est de montrer comment il m'a été possible d'appliquer aux cours de formation en interprétation plusieurs théories tirées de la psychologie cognitive ainsi que de la neurologie.

Après avoir travaillé comme assistante de recherche pour Mme Patricia $\mathbf{E}$. Longley à la Polytechnique de Londres (1976-1979), enseigné la traduction et l'interprétation au Monterey Institute of International Studies, en Californie (1979-1984), et à l'Ecole de traducteurs et d'interprètes à l'Université d'Ottawa (1984 à ce jour), j'ai pu appliquer certains aspects du traitement de l'information à l'interprétation simultanée et structurer mon séminaire autour des objectifs d'apprentissage suivants :

1) l'écoute et la rétention

2) le «shadowing»

3) le traitement en parallèle (ou «dual-task training»)

4) la paraphrase

5) la contraction de texte (ou «abstracting»)

6) l'exercice de closure

7) la traduction à vue

8) l'interprétation à vue

9) le traitement de chiffres, d'acronymes et de noms propres

10) le décalage

11) l'anticipation

12) la latéralisation ou dominance cérébrale.

Avant de décrire chacun de ces objectifs, $\mathrm{j}$ 'aimerais préciser que je ne suis pas de celles qui pensent que la meilleure façon d'enseigner la natation à un enfant est de le jeter carrément à l'eau et de voir comment il se débrouille. Si je peux comparer l'interprétation simultanée à une tâche motrice comme la conduite automobile, j'oserai encore moins prétendre que la meilleure façon d'apprendre à conduire est de s'asseoir au volant d'une automobile et de faire du 100 à l'heure sur l'autoroute. Pour apprendre à bien conduire, il est plus logique, me semble-t-il, de maitriser une activité avant de passer à la suivante: je commencerais tout d'abord, bien entendu, par faire démarrer l'auto, puis je laisserais réchauffer le moteur, j'apprendrais à changer de vitesse sans pour autant faire avancer la voiture avant de m'aventurer sur la route. De même, pour initier les débutants à l'interprétation simultanée, je procède par étapes.

Certains des exercices que je propose peuvent servir de tests de sélection afin de prédire quels candidats risquent d'échouer ou quels sont ceux qui ont une chance, au contraire, de réussir l'examen de sortie à la fin d'un programme de formation d'interprètes ${ }^{1}$. De plus, les exercices en question servent aussi de tests éliminatoires; un étudiant, par exemple, ne pourrait pas passer à l'étape de l'apprentissage de l'interprétation simultanée tant qu'il n'aurait pas appris à maîtriser le «shadowing». 
Les exercices sont présentés dans un ordre chronologique, du plus simple au plus complexe suivant un enchaînement progressif. Ils se rapprochent le plus possible de l'interprétation simultanée. Certains exercices exigeront plus de temps à maîtriser que d'autres et les étudiants devront comprendre dès le départ que tout exercice présenté par le chargé de cours en laboratoire, demandera, de leur part, au moins trois ou quatre répétitions faites à la maison. Pour terminer, je dois aussi préciser que mes douze objectifs ne représentent en aucune façon un programme d'études complet; il s'agit tout simplement d'un cours d'initiation à l'interprétation simultanée pouvant s'étendre sur un ou deux trimestres.

\section{1) L'ÉCOUTE ET LA RÉTENTION}

Le but de cet exercice est de déterminer la capacité de compréhension de l'étudiant débutant, lors de l'écoute, et sa capacité de rétention après l'écoute. L'écoute et la compréhension sont présentes dans toutes les tâches requises d'un interprète. Pour ce faire, on demande à l'étudiant d'écouter passivement (c'est-à-dire sans prendre de notes) un texte relativement court, présenté soit viva voce soit sur cassette, tout en le prévenant qu'il devra par la suite essayer de se souvenir des éléments d'information importants de l'extrait entendu.

On pourra objecter que les interprètes de conférence ne sont jamais appelés à se souvenir du texte qu'ils viennent d'interpréter et que par conséquent, ils prennent des notes. C'est exact, mais le but de cet exercice est de déceler le plus tôt possible chez l'apprenti interprète sa capacité de rendre le plus fidèlement possible un message sans en déformer le sens, sans broder, sans oublier les points importants.

En outre, l'utilité de cet exercice est multiple. Il permet, entre autres, de vérifier le degré de compétence d'un sujet selon qu'il écoute un passage présenté dans sa langue maternelle (L1) ou sa deuxième langue (L2). L'exercice peut donc prendre les quatre formes suivantes :

1) l'écoute en $L I$ avec rétention en $L I$ (même langue)

2) l'écoute en $L 2$ avec rétention en $L 2$ (" ")

3) l'écoute en $L 2$ avec rétention en $L 1$ (traduction)

4) l'écoute en $L 1$ avec rétention en $L 2$ (" ")

Examinons l'hypothèse suivante: un candidat réussit le premier volet, où tout se déroule dans sa langue maternelle, mais échoue lamentablement au troisième volet, où l'on introduit la traduction (transfert linguistique) comme variable. Cet étudiant comprendrait qu'il a une bonne mémoire (test 1), mais que sa maîtrise passive ou sa compréhension de sa deuxième langue, ou l'une et l'autre, sont insuffisantes (test 2). Il pourrait donc centrer ses efforts sur le perfectionnement de sa langue passive avant de passer à l'étape suivante. Comme tout candidat a le droit de se présenter deux fois aux tests de sélection de notre école, nous aimons leur indiquer leurs faiblesses dès le début pour qu'ils sachent ainsi sur quel aspect faire porter leur attention. Cela vaut mieux, croyons-nous, que de les recaler sans leur fournir d'explications.

\section{2) LE «SHADOWING»}

L'activité qui se rapproche «mécaniquement» le plus de l'interprétation simultanée (c'est-à-dire parler et écouter en même temps), est le shadowing ou répétition simultanée d'un message. Colin Cherry (1953) décrit le shadowing comme la répétition à haute voix d'un message en prose parlée, répétition dans laquelle on tente de rester aussi proche que possible des paroles originales. Cela est relativement facile si le débit du message n'est pas trop rapide et le nombre d'erreurs est généralement minime (Neisser, 1966). Si cette activité n'est pas fréquente dans la vie courante, elle trouve néanmoins des applications 
en psychologie expérimentale, et elle s'est révélée fort utile dans les études sur l'attention divisée et l'écoute sélective (voir, par exemple, les travaux de Cherry, 1953; Broadbent, 1958, et Treisman, 1964). Elle permet en effet d'explorer les limites de traitement de l'information et la capacité humaine à exécuter simultanément deux ou plusieurs tâches. Mais le shadowing est aussi une excellente technique de formation des interprètes, dans la mesure où un débutant doit d'abord s'habituer à pouvoir exécuter deux tâches simultanément (écoute et production verbale) dans la même langue (shadowing) avant d'exécuter ces deux mêmes tâches dans deux langues différentes (interprétation simultanée).

Comme le fait observer Miller (1963), il est exceptionnel qu'un individu ait un comportement linguistique dans lequel il émet une production verbale et en écoute une autre simultanément; généralement, l'écoute et la production verbale sont consécutives; un individu écoute un message et attend une pause dans le débit de l'interlocuteur pour prendre à son tour la parole. De plus, selon Barik (1973), tous les interprètes ne pouvant maîtriser l'écoute et la production verbale simultanées ont tendance à développer de mauvaises habitudes dès le départ; ils prennent pour ainsi dire un mauvais pli. Par exemple, beaucoup de débutants en interprétation essaient, consciemment ou inconsciemment, de parler le moins possible en même temps que le délégué, mais le plus possible durant les pauses de fin de phrase. Le débit de l'interprète est donc inégal, saccadé, ce qui révèle un manque de professionalisme. L'hypothèse selon laquelle l'interprète simultané fait un usage maximal des pauses dans le discours en langue-source afin de réduire le temps pendant lequel il doit écouter et produire en même temps a été avancée par Goldman-Eisler (1968) et vérifiée par Henri Barik (1973). Si ce dernier a pu démontrer que l'interprète fait une utilisation accrue des pauses dans la langue-source, il n'a toutefois pas pu établir de corrélation positive entre l'utilisation des pauses et la qualité de l'interprétation. Gerver (1976) note que des pauses d'une à deux secondes ne laissent pas le temps de prononcer beaucoup de paroles. Gerver $(1972 a ; 1972 b)$ arrive à la double conclusion suivante: d'une part, les pauses en langue-source sont essentiellement de courte durée et, d'autre part, très peu de production verbale en langue-cible peut y être intercalée. La difficulté de l'interprétation simultanée réside précisément dans le fait qu'une grande partie de l'écoute et de la production verbale en langue-cible se fait simultanément.

Certains psychologues (Norman, 1976) font la distinction entre deux formes de shadowing, les deux pouvant servir d'exercices pour la formation des interprètes. Le shadowing phonémique, où le sujet répète ce qu'il entend dès la première syllabe, tout en essayant de rester le plus près possible de la langue source. Le shadowing de phrase, où le sujet répète ce qu'il entend avec un décalage de plus de 250 millisecondes par rapport à la langue source. Autrement dit, comme l'interprète, le sujet dans ce cas commence à répéter ce qu'il entend à la suite d'une proposition, ou d'une unité de sens tel un nom + sujet + objet direct. Une étude menée par Chistovitch, Aliakrinskii et Abilian (1960) a d'ailleurs démontré que les personnes qui pratiquaient le shadowing avec décalage avaient une meilleure rétention du sujet traité que celles qui optaient pour le shadowing phonémique avec un minimum de décalage.

Il est recommandé d'utiliser les deux versions du shadowing avec les débutants en interprétation. On commencera, bien sûr, par le shadowing phonémique afin de vérifier si le candidat interprète maitrise facilement l'écoute et la production verbale simultanées. Ensuite, on passera au shadowing phrastique, en augmentant le décalage afin de l'habituer progressivement à attendre avant de commencer à parler. Tout étudiant ne maîtrisant pas le premier volet de l'exercice devrait le perfectionner avant de passer au suivant.

Les exercices du shadowing peuvent aussi présenter des défis de plus en plus intéressants. Par exemple, le professeur peut lire le texte-source de plus en plus vite afin d'habituer l'apprenti interprète au stress causé par un débit dépassant 150 mots/minute. 
De plus, on peut lui demander de faire du shadowing à partir de discours prononcés par un orateur ayant un fort accent ou encore amplifier les bruits de fond.

\section{3) LE TRAITEMENT EN PARALLÈLE OU «DUAL-TASK TRAINING»}

Il serait hors de notre propos de résumer tout ce que la psychologie expérimentale et cognitive nous révèle sur la capacité d'exécuter deux tâches simultanément. Retenons cependant que cette capacité d'exécution semble peu naturelle et exige énormément des facultés humaines permettant de traiter de l'information, en particulier lorsqu'il s'agit d'écouter et de parler simultanément (Broadbent, 1952). Notons également que la difficulté d'accomplir deux tâches simultanées augmente dans la mesure où celles-ci font appel à des mécanismes d'analyse semblables; bien que cela soit difficile, ce n'est pas une chose impossible et l'on peut s'y rendre habile par un apprentissage (Hamers et Blanc, 1983). La capacité de l'interprète d'effectuer deux tâches simultanément implique que tout modèle de traitement de l'information doit prévoir un mécanisme permettant soit d'emmagasiner l'information simultanément, soit de la traiter parallèlement. Treisman (1969), par exemple, a suggéré que deux informations peuvent être traitées en parallèle à condition de ne pas faire appel au même mécanisme d'analyse. Selon Neisser (1966), ce traitement parallèle se fait au niveau préattentif, ce qui permet d'emmagasiner ou de rejeter l'information ne pouvant être traitée immédiatement. C'est à ce niveau préattentif que se situerait l' «automatisme». Par «automatisme», on entend le fait que la réponse surgit spontanément, alors que l'analyse du stimulus, elle, a lieu à un niveau moins profond d'analyse ; dans ce dernier cas, l'effort mental est moindre et n'exige plus autant d'attention, celle-ci pouvant alors se porter sur une autre tâche.

Tout cela peut faire l'objet d'un apprentissage. Au Monterey Institute of International Studies, le professeur Barbara Moser-Mercer, passée depuis à l'ETI de Genève, initiait les étudiants à effectuer ce traitement en parallèle de l'information de la façon suivante: partant du principe de base que tout interprète doit d'abord apprendre à écouter, Moser demandait à ses étudiants d'écouter attentivement un texte enregistré au préalable (tâche A), tout en les prévenant qu'ils allaient devoir s'en souvenir par la suite. En même temps, elle leur demandait de compter à rebours, (tâche B), à haute voix, des chiffres impairs, de 99 à 1, par exemple. Peu à peu, le fait de compter à haute voix, devenait de plus en plus «automatique» et les étudiants apprenaient ainsi à traiter de l'information parallèlement tout en dirigeant leur attention vers la tâche exigeant le plus de concentration, celle de l'écoute.

\section{4) LA PARAPHRASE}

Une fois acquis le shadowing et le traitement de l'information en parallèle, il est temps de passer à la paraphrase. Encore une fois, il serait hors propos de résumer ici toutes les recherches effectuées en linguistique sur les vertus didactiques de la paraphrase. Du point de vue du traitement de l'information chez l'être humain, la paraphrase nous rapproche davantage de l'interprétation simultanée, en ce sens que toute traduction n'implique que très rarement une reformulation mot-à-mot. Traduire consiste plutôt à paraphraser jusqu'à un certain point l'original.

Tout débutant en interprétation simultanée a tendance non seulement à trop «coller» à l'original, mais aussi à traduire mot-à-mot. Les exercices de paraphrase, en début de formation, visent à remédier à ce défaut. Pour y arriver, on demande à l'étudiant de reformuler le message original, dans la même langue, (sinon, ce serait de l'interprétation) en n'utilisant pas les mêmes termes que ceux de l'original (paraphrase lexicale), tout en essayant de transformer l'ordre de la phrase (paraphrase syntaxique).

Une fois de plus, comme pour les exercices du shadowing, on peut augmenter le degré de difficulté des exercices en choisissant des textes plus ou moins denses ou plus 
ou moins techniques, en faisant varier la rapidité du débit de la langue-source, en utilisant des enregistrements d'interlocuteurs ayant des accents divers, et ainsi de suite.

Le professeur Moser (1983) a observé que les candidats peu doués pour la paraphrase obtenaient généralement les notes finales les plus basses de son cours d'initiation à l'interprétation simultanée, à Monterey. Selon elle, la paraphrase serait un test assez fiable pour sélectionner les meilleurs interprètes.

\section{5) LE RÉSUMÉ DE TEXTE OU «ABSTRACTING»}

Cet exercice consiste à résumer les idées principales d'un texte ou d'un discours en éliminant tous les détails superflus, comme les propositions subordonnées, les relatives, les appositions, les adjectifs, etc. Seules sont retenues les propositions principales. $\mathrm{Ce}$ résumé diffère de la contraction de texte au sens propre du terme. Il s'agit d'un exercice conçu par Ine van Dam (1986) au Monterey Institute of International Studies. Dans son document de travail, van Dam propose plusieurs exercices de résumé grâce auxquels les étudiants peuvent apprendre à rattraper un orateur en cas de blocage et à revenir au décalage optimal, sans pour autant escamoter des bouts de phrase.

Comme premier exercice, van Dam propose un résumé consécutif: l'étudiant écoute une phrase, sans prendre de notes, et une fois la phrase énoncée, il la résume dans la même langue. Une pause permet à l'étudiant d'énoncer son résumé.

Un deuxième exercice consiste à reprendre les phrases du premier exercice, mais à supprimer les pauses entre chacune d'elles. L'étudiant a donc, comme en interprétation simultanée, à écouter une phrase pendant qu'il résume la précédente. La différence est que le résumé se fait dans la même langue.

Pour le troisième exercice, on reprend les mêmes phrases et on demande à l'étudiant de les interpréter. L'étudiant qui a entendu les mêmes phrases trois fois de suite y arrive généralement du premier coup. Dans le cas contraire, l'auteur recommande qu'il applique la technique du résumé pour rattraper l'orateur dans la langue source et ainsi revenir à un décalage normal.

\section{6) L'EXERCICE DE CLOSURE}

Le procédé de closure consiste à éliminer des mots dans un texte de prose, par exemple tous les dix mots, et à demander à un sujet de combler les trous. Cette technique a été employée fréquemment lors des recherches sur le comportement linguistique et cognitif des sujets bilingues. Selon Stubbs et Tucker (1974), l'exercice de closure est un des outils les plus précis pour les spécialistes de l'enseignement d'une langue seconde et une méthode pédagogique de grande valeur pour l'enseignant de cette langue. En effet, l'exercice de closure s'est avéré un des moyens les plus sûrs pour vérifier la compétence linguistique d'un individu, car il permet de tenir compte des aspects lexicaux, syntaxiques et sémantiques du traitement du langage chez l'être humain.

L'un des aspects fondamentaux de la compétence linguistique est la capacité d'anticiper des éléments sur une chaîne linéaire, surtout quand ces éléments sont présentés à une grande vitesse dans un temps donné. Oller (1972) a trouvé que l'exercice de closure était un bon instrument de mesure de cette compétence chez l'être humain. Le test de closure a aussi des usages multiples: on peut le présenter à un sujet dans sa langue maternelle, puis dans sa deuxième langue. Plus un sujet a une connaissance approfondie de sa langue seconde, plus il lui sera facile de remplacer les mots manquants dans le texte de départ.

Stubbs et Tucker (1974) expliquent comment élaborer et corriger le test de closure. Ils recommandent, en bref, de laisser les premières phrases intactes afin de fournir un contexte sémantique au sujet. On peut omettre les mots à intervalle régulier, tous les dix 
mots, par exemple, comme le recommande Oller (1972) et Stubbs et Tucker (1974), ou supprimer sélectivement certains mots afin de mesurer les connaissances générales du sujet (Lambert, 1987). À l'Université d'Ottawa, par exemple, nous demandons à nos étudiants de lire les journaux quotidiennement afin qu'ils se tiennent au courant de l'actualité politique à l'échelle nationale et internationale. Le test de closure peut donc être choisi en fonction de cet objectif. Le texte d'illustration ci-dessous a été publié au moment de la vague d'attentats terroristes à Paris.

Texte A (avec suppression d'un mot, tous les dix mots):

\section{The Bombs of September}

Wednesday is a busy day for the Tati discount (store) on the Rue de Rennes in Paris. School is (out) that afternoon, and mothers, particularly those with modest incomes, (flock) to Tati with their children in search of bargains. (Thus) the sidewalk in front of the store was bustling 5:28 p.m., when two black-mustachioed men in (a) black BMW drove past.

Texte B (évaluation des connaissances générales, richesse de vocabulaire, mémoire):

\section{The Bombs of September}

Wednesday is a busy day for the discount store on the Rue de Rennes in School is that afternoon, and mothers, particularly those with incomes, flock to Tati with their children in search of sidewalk in front of the store was bustling last week at 5:28 p.m., when Thus the mustachioed men in a black. down, the man on the drove past. As the car blackger) side got out and dropped a (slowed) age) into a trash can near the front door. He quickly rode off. A few (seconds) later, an explosion shattered the happy (sounds) of shoppers. "There was simply a noise, very loud, then the of the people", recalled a (witness).

(Time, September 29, 1986)

Dans le cas du premier texte, le test de closure ne mesure que la compétence linguistique d'un individu. Dans le second cas, où les mots sont omis en fonction des objectifs d'apprentissage chez les étudiants en interprétation, le test ne mesure pas uniquement leur connaissance d'une seconde langue, mais aussi leur connaissance de l'actualité. Le texte B vérifie donc si l'étudiant a relevé le nom du magasin Tati et s'il se souvient du fait que ces événements ont eu lieu à Paris, et non en Irlande du Nord, par exemple.

De plus, le texte $B$ mesure aussi la richesse de vocabulaire de l'étudiant dont l'anglais n'est pas nécessairement la langue maternelle. Soit la phrase «dropped a pack$a g e »$. Le synonyme «parcel» aurait été acceptable, mais le choix de «bomb» aurait été fautif, car n'oublions pas que le colis ou le paquet en question avait été enveloppé de façon à dissimuler la bombe. Il en est de même pour la phrase «the man on the passenger side...», où seul un sujet ayant une maîtrise quasi parfaite de l'anglais réussirait à trouver le mot juste: le choix de «left» ou de «right side» serait inacceptable. 
Le texte $B$ peut aussi servir à tester les connaissances générales des étudiants. Combien de Nord-Américains savent que le mercredi est jour de congé pour les écoliers en France?

Finalement, le deuxième texte nous renseigne aussi sur la précision de la mémoire, sur l'aptitude des candidats à retenir des détails aussi précis que le fait que deux et non pas trois individus ont perpétré l'attentat de la rue de Rennes et que ces deux terroristes étaient à l'avant d'une BMW noire. Des détails, bien sûr, mais tout interprète chevronné sait que des détails comme ceux-là ont leur importance.

Le test de closure recèle d'énormes possibilités. À titre d'exemple, John Long, de Cambridge, a eu l'idée de l'adapter afin de le présenter non pas visuellement, comme je viens de le montrer avec les textes $\mathrm{A}$ et $\mathrm{B}$, mais sur support auditif, c'est-à-dire sur cassette. Pour ce faire, le texte est lu et enregistré sur cassette et le mot à omettre est remplacé par une tonalité indiquant au sujet qu'il y a là un mot à remplacer. Les mots peuvent être reconstitués de la façon suivante:

a) On peut demander au sujet d'écouter le texte et d'écrire les mots manquants sur une feuille de papier (Gerver, Longley, Long, et Lambert, 1984).

b) On peut demander au sujet d'écouter le texte et de prononcer à voix haute, les mots manquants.

c) On peut ensuite demander aux étudiants en formation de faire un exercice de shadowing avec un texte de closure et de combler les trous.

d) Finalement, on peut demander aux étudiants d'interpréter en simultanée un texte de closure et de combler les trous au fur et à mesure. Dans mes cours, je commence par lire le texte en entier, intact, et je demande aux étudiants de le répéter dans la même langue, en respectant ma tonalité et la vitesse à laquelle je le lis. Dans un deuxième temps, je relis le même texte mais j'omets certains mots, comme dans le cas du texte $\mathrm{B}$, afin de tester le niveau de compétence linguistique des étudiants, leurs connaissances générales, la richesse de leur vocabulaire et leur capacité de rétention.

\section{7) LA TRADUCTION À VUE}

La traduction à vue est la transposition d'un texte écrit dans une langue en un texte prononcé à haute voix dans une autre langue. Puisqu'il s'agit d'un traitement d'information visuelle et orale, la traduction à vue est parfois comparée à l'interprétation simultanée (Moser, communication personnelle). Leurs points communs sont, entre autres, les restrictions temporelles (si la traduction à vue est considérée comme une épreuve contre la montre), l'anticipation (savoir lire jusqu'à la fin de la phrase avant d'amorcer la traduction), la nature orale de la traduction à vue et, enfin, le stress causé par la présence du chargé de cours et des autres étudiants dans la classe. La traduction à vue peut se faire avec ou sans préparation : à Monterey, par exemple, lors des examens de sortie, les candidats font leurs traductions à vue ( $L 3$ vers $L 1, L 2$ vers $L 1$, et $L 1$ vers $L 2$ et vice-versa pour les bilingues ou ceux qui ne travaillent que dans deux langues) sans préparation, sans même avoir le temps de lire le texte.

\section{8) L'INTERPRÉTATION À VUE}

Le programme en interprétation de l'Université d'Ottawa vient de s'enrichir d'un nouveau volet: l'interprétation à vue, la raison étant que la majorité de nos diplômés postulent un emploi au Secrétariat d'État où l'examen de sélection comprend un test d'interprétation à vue. Nous donnons aux étudiants un texte qu'ils peuvent étudier pendant cinq minutes. Nous leur recommandons de profiter au maximum du temps alloué pour bien lire le texte du début à la fin au lieu de s'attarder uniquement sur le premier paragraphe. Nous leur conseillons aussi de souligner les mots difficiles, les noms propres, ou tout terme susceptible de bloquer l'interprète. 
Quand les cinq minutes sont écoulées, le candidat passe en cabine et se prépare, avec le texte devant lui, à interpréter une voix sur cassette, qui lit le texte en question.

Il arrive souvent qu'un interprète ne reçoive la documentation nécessaire que quelques minutes avant le début de la conférence. Aussi, dans nos cours à l'Université d'Ottawa, quand nous lisons un texte que l'étudiant a devant lui, nous faisons comme tout délégué : nous lisons certains paragraphes, nous brodons sur un point un peu confus, nous sautons des portions de phrase, etc. L'étudiant s'habitue peu à peu à l'interférence visuelle produite par le texte; il apprend à se détacher graduellement du mot-à-mot et à ne jamais devancer l'orateur.

\section{9) L'INTERPRÉTATION DE CHIFFRES, D'ACRONYMES, ET DE NOMS PROPRES}

Des textes utilisés dans ces exercices doivent contenir évidemment le plus de chiffres, de statistiques, de noms propres et d'acronymes possible. Souvent, il faut les «bricoler» soi-même. Les étudiants en cabine s'exercent soit individuellement, en notant les chiffres au fur et à mesure qu'ils les entendent, soit à deux, l'un écrivant les chiffres tandis que l'autre interprète le texte tout en lisant les chiffres transcrits par le premier.

\section{0) LE DÉCALAGE}

Dans son manuel Simultaneous Interpreting: A Practice Book, Paul Hendrickx (1971) propose une série d'exercices permettant au chargé de cours de contrôler et ainsi de mesurer le décalage chez les apprentis interprètes. Les exercices comprennent des pages de mots, d'expressions, de phrases courtes, le tout présenté dans un ordre progressif allant du plus simple au plus complexe. L'enchaînement se fait donc en commençant par des mots 1) élémentaires, 2) généraux, 3) économiques, 4) scientifiques et techniques, et 5) de terminologie de conférence. Après les unités lexicales, viennent les phrases courtes ne comprenant qu'un sujet, un verbe, et un complément d'objet direct.

On demande à l'étudiant soit de répéter, soit d'interpréter des mots, d'abord avec un décalage d'un seul mot, puis avec un décalage plus long, l'écart étant de 1 à 5 mots ou plus, selon la capacité de chacun. Cet exercice oblige les sujets à retenir en mémoire de plus en plus de termes, ce qui leur permet de trouver leur décalage optimal.

\section{1) L'ANTICIPATION}

Comme pour l'exercice de closure décrit plus haut, les exercices d'anticipation obligent les étudiants à terminer une phrase en tenant compte du contexte. Pour ce faire, le professeur lit un passage ou fait jouer une cassette et interrompt le message en pleine phrase. Il demande aux étudiants de terminer la phrase le plus logiquement possible.

\section{2) LA LATÉRALITÉ OU DOMINANCE CÉRÉBRALE}

Les étudiants sont invités, dès le premier cours, à essayer de trouver la condition optimale pour l'écoute: certains interprètes préfèrent travailler casqués d'écouteurs; d'autres, au contraire, préfèrent écouter le message à interpréter d'une oreille tout en s'écoutant de l'autre. Dans ce cas, une oreille est recouverte, l'autre ne l'est pas.

Je mène présentement une étude sur l'oreille gauche et sur l'oreille droite en interprétation simultanée. Il semblerait que les interprètes droitiers préfèrent se servir de l'oreille gauche pour écouter le message à interpréter, tandis que la droite leur servirait davantage à s'écouter eux-mêmes (Lambert, à paraître).

\section{NOTE}

1. Plusieurs des objectifs cités ci-dessus servent déjà de tests de sélection à l'Université d'Ottawa. Ils sont expliqués plus en détail dans trois publications : Gerver, Longley, Long \& Lambert, 1984 ; Lambert \& Meyer, 1988 ; Lambert, à paraître. 


\section{BIBLIOGRAPHIE}

BARIK, H.C. (1973) : Simultaneous Interpretation: Temporal and Quantitative Data, Language and Speech, 16, pp. 237-270.

BROADBENT, D.E. (1952) : Speaking and Listening Simultaneously, Journal of Experimental Psychology, 43, pp. 267-273.

BROADBENT, D.E. (1958) : Perception and Communication, London : Pergamon Press.

CHERRY, C. (1953): Some Experiments on the Recognition of Speech With One and Two Ears, Journal of the Acoustic Society of America, 5, pp. 975-979.

CHISTOVITCH, L.A., ALIAKRINSKII, V.V. \& ABILIAN, V.A. (1960) : Time Delays in Speech Perception, Questions of Psychology, 1, pp. 64-70.

GERVER, D. (1972a): A.S.P.A.: Automatic Speech-Pause Analyzer, Behavioral Research Methods and Instrumentation, 4, pp. 265-270.

GERVER, D. (1972b): Simultaneous and Consecutive Interpretation and Human Information Processing, London: Social Science Research Council Report HR566-1.

GERVER, D. (1976) : Empirical Studies of Simultaneous Interpretation: A Review and A Model, in R.W. Brislin (ed.), Translation: Application and Research, New York : Gardner Press, pp. 165-207.

GERVER, D., LONGLEY, P., LONG, J. \& LAMBERT, S. (1984): Selecting Trainee Conference Interpreters : A Preliminary Report, Journal of Occupational Psychology, 57, 1, pp. 17-31.

HAMERS, J.F. \& BLANC, M. (1983) : Bilingualité et bilinguisme, Editions Pierre Margada, Bruxelles.

HENDRICKX, P.V. (1971) : Simultaneous Interpreting: A practice Book, Longman : London.

LAMBERT, S. M. (1987): The Cloze Technique As a Pedagogical Tool for the Training of Translators and Interpreters, Jerome Quarterly, 2, 2, 13 .

LAMBERT, S.M.: Proficiency of Interpretation According to Ear of Input Information, Brain and Language, (in press).

LAMBERT, S.M. : Selection Tests for Trainee Conference Interpreters, META, (in press).

LAMBERT, S.M. \& MEYER, I. (1988) : Selection Examinations For Student Interpreters at the University of Ottawa, Canada, Canadian Modern Language Review, 44, 2, pp. 274-284

MILLER, G. A. (1963): Review of J.H. Greenberg: Universals of Language, Contemporary Psychology, 8, pp. 81-83.

MOSER, B. (1983) : Testing Interpreting Aptitude, in Wolfram Wilss and Gisela Thome (Eds.), Translation Theory and Its Implementation in the Teaching of Translating and Interpreting, Association internationale de linguistique appliquée, Saarbrucken, juillet 25-30, pp. 318-325.

NEISSER, U. (1966) : Cognitive Psychology, New York : Appleton-Century-Croft.

NORMAN, D.A. (1976) : Memory and Attention, New York: Wiley.

OLLER, J.W. (1979) : Language Tests at School, London : Longman.

STUBBS, J.B., \& TUCKER, G.R. (1974): The Cloze Test As a Measure of English Proficiency, Modern Language Journal, 58 , pp. 239-241.

TREISMAN, A.M. (1964) : Monitoring and Storage of Irrelevant Messages in Selective Attention, Journal of Verbal Learning and Verbal Behavior, 3, pp. 449-459.

TREISMAN, A.M. (1969) : Strategies and Models of Selective Attention, Psychological Review, 76, pp. 282-299.

VAN DAM, I. (1986): Strategies of Simultaneous Interpretation: A Methodology for the Training of Simultaneous Interpreters, Mimeographed Report, Monterey Institute of International Studies. 